\title{
CONVERGENCE TO SELF-SIMILARITY IN AN ADDITION MODEL WITH POWER-LIKE TIME-DEPENDENT INPUT OF MONOMERS
}

\author{
F.P. DA COSTA \\ Universidade Aberta, DCET \\ Rua Fernão Lopes 9, 2º Dto, P-1000-132 Lisboa, Portugal \\ and \\ Universidade Técnica de Lisboa, Instituto Superior Técnico, CAMGSD \\ Av. Rovisco Pais 1, P-1049-001 Lisboa, Portugal \\ JOÃO T. PINTO \\ Universidade Técnica de Lisboa, Instituto Superior Técnico, \\ Departamento de Matemática and CAMGSD \\ Av. Rovisco Pais 1, P-1049-001 Lisboa, Portugal \\ RAFAEL SASPORTES \\ Universidade Aberta, DCET \\ Rua Fernão Lopes 9, 20 Dto, P-1000-132 Lisboa, Portugal
}

\begin{abstract}
In this note we extend the results published in Ref. 1 to a coagulation system with Becker-Döring type interactions and time-dependent input of monomers $J_{1}(t)$ of power-like type: $J_{1}(t) /\left(\alpha t^{\omega}\right) \rightarrow 1$ as $t \rightarrow \infty$, with $\alpha>0$ and $\omega>-\frac{1}{2}$. The general framework of the proof follows Ref. 1 but a different strategy is needed at a number of points.

Keywords: Dynamics of non-autonomous ODEs, Coagulation equations, Longtime behaviour, Self-similar behaviour.
\end{abstract}

\section{Introduction}

There has been a recent upsurge of mathematical work in the field of coagulation-type equations (see, for instance, References 2 and 3, and references therein) of which a sizable portion has been dedicated to dynamical questions, particularly those of convergence to self-similar behaviour (see the references above and also Ref. 4). In the current note we consider this type of problems in the context of the following addition model 
(i.e., a coagulation equation with Becker-Döring type interactions) with time-dependent input of monomers, $J_{1}(t)$. Calling a cluster with $j$ identical particles a $j$-cluster, and a single particle a 1 -cluster, or a monomer, the kinetic scheme associated with the above mentioned process of cluster growth is

$$
j \text {-cluster }+1 \text {-cluster } \longrightarrow(j+1) \text {-cluster, } \quad j \geq 1 \text {. }
$$

We assume the existence of a monomer source, possibly time-dependent. Assuming also that the mass action law of chemical kinetics is valid, and denoting by $c_{j}=c_{j}(t)$ the concentration of $j$-clusters at time $t$, the following system of ordinary differential equations describes the time evolution of the concentrations $c_{j}$ :

$$
\left\{\begin{array}{l}
\dot{c}_{1}=J_{1}(t)-c_{1}^{2}-c_{1} \sum_{j=1}^{\infty} c_{j} \\
\dot{c}_{j}=c_{1} c_{j-1}-c_{1} c_{j}, \quad j \geq 2 .
\end{array}\right.
$$

Systems of this type are used, for instance, as mean-field models of submonolayer growth in epitaxial deposition (cf., e.g., Ref. 5).

This work is a follow-up study to Ref. 1 and the reader should consult that paper for a more comprehensive explanation of the background and motivation involved. In Ref. 1 monomer input terms $J_{1}(t)=\alpha t^{\omega}$, with $\alpha>0$ and $\omega>-\frac{1}{2}$, were considered. This is clearly a rather restrictive class, and here we provide an extension of those results to monomer input of the type $J_{1}(t)=\alpha t^{\omega}(1+\varepsilon(t))$ where $\varepsilon(\cdot)$ is a continuous function satisfying $\varepsilon(t) \rightarrow 0$ as $t \rightarrow+\infty$. Most, but not all, of the results in Ref. 1 follow with just this general assumption on $\varepsilon$. This is true, in particular, of those concerning convergence to a self-similar profile. Those about the rate of convergence of the bulk quantity $\sum_{j=1}^{\infty} c_{j}$ will need an extra assumption on the decay rate of the perturbation $\varepsilon(t)$.

\section{General Approach and Statement of Results}

Our main goal is to study the long-time behaviour and approach to selfsimilarity of solutions to Equation (1). The general approach, used also in Refs. 6 and 1 is to consider the auxiliary variable $c_{0}(t)=\sum_{j=1}^{\infty} c_{j}(t)$, representing the total amount, at time $t$, of clusters of every possible size $j$. This quantity satisfies the differential equation $\dot{c}_{0}=J_{1}(t)-c_{0} c_{1}$, and so 
(1) can be written as

$$
\left\{\begin{array}{l}
\dot{c}_{0}=J_{1}(t)-c_{0} c_{1} \\
\dot{c}_{1}=J_{1}(t)-c_{0} c_{1}-c_{1}^{2} \\
\dot{c}_{j}=c_{1} c_{j-1}-c_{1} c_{j}, \quad j \geq 2
\end{array}\right.
$$

From this we conclude firstly that the dynamic behaviour of the monomer concentration $c_{1}(t)$ is determined only by the bidimensional $\left(c_{0}, c_{1}\right)$-system,

$$
\left\{\begin{array}{l}
\dot{c}_{0}=J_{1}(t)-c_{0} c_{1} \\
\dot{c}_{1}=J_{1}(t)-c_{0} c_{1}-c_{1}^{2}
\end{array}\right.
$$

and, secondly, after introducing the new time scale $t \longmapsto \varsigma(t):=\varsigma_{0}+$ $\int_{t_{0}}^{t} c_{1}(s) d s$, the dynamics of the $j$-cluster becomes determined by the (infinite dimensional) lower triangular linear equations

$$
\widetilde{c}_{j}{ }^{\prime}=\widetilde{c}_{j-1}-\widetilde{c}_{j}, \quad j \geq 2,
$$

where $\widetilde{c}_{j}(\varsigma):=c_{j}(t(\varsigma))$ and $(\cdot)^{\prime}=\frac{d}{d \varsigma}$, which can be explicitly solved in terms of $\widetilde{c}_{1}$ by a repeated application of the variation of constants formula:

$$
\widetilde{c}_{j}(\varsigma)=e^{-\varsigma} \sum_{k=2}^{j} \frac{\varsigma^{j-k}}{(j-k) !} c_{k}(0)+\frac{1}{(j-2) !} \int_{0}^{\varsigma} \widetilde{c}_{1}(\varsigma-s) s^{j-2} e^{-s} d s .
$$

We shall consider the following general assumption for the input of monomers $J_{1}(t)$ :

(H1) $J_{1}(t)=(1+\varepsilon(t)) \alpha t^{\omega}$, where $\alpha>0, \omega>-\frac{1}{2}$, and $\varepsilon(t)$ is a continuous function satisfying $\varepsilon(t) \rightarrow 0$ as $t \rightarrow+\infty$.

The case where $\varepsilon(t) \equiv 0$ was considered in Ref. 1 and the particular case where, additionally to that, we had time independent input $\omega=0$ was first studied in Ref. 6. Both these studies contributed to the rigorous justification of formal results in Ref. 7. The case of polynomial-like monomer input term considered in this note has never been investigated before, not even at a formal level.

The main idea behind our approach is that, since $\varepsilon(t) \rightarrow 0$ as $t \rightarrow+\infty$, the presence of this perturbation term should not be felt at large times, and so the results in Ref. 1 should remain valid. In particular, we should expect the following to hold true:

Theorem 2.1. Assume (H1) holds, and let $\left(c_{0}, c_{1}\right)$ be any solution of Eq. (3). Then, as $t \rightarrow+\infty$, we have 
(i) $\left(\frac{1+2 \omega}{3 \alpha^{2}}\right)^{\frac{1}{3}} t^{-\frac{1+2 \omega}{3}} c_{0}(t) \longrightarrow 1$,

(ii) $\left(\frac{3}{(1+2 \omega) \alpha}\right)^{\frac{1}{3}} t^{\frac{1-\omega}{3}} c_{1}(t) \longrightarrow 1$.

Using the result in Theorem 2.1 (ii) and translating it to the phase variable $\widetilde{c}_{1}$ in the time scale $\varsigma$ we can use it in Eq. (5) to obtain information on the long time behaviour of the $j$-cluster concentration $\widetilde{c}_{j}(\varsigma)$, in particular concluding that the behaviour in Theorem 2.1 (ii) holds true with $c_{1}(t)$ substituted by $c_{j}(t)$, for every $j \geq 1$. Furthermore, and more interesting from a dynamical point of view, the information about $\widetilde{c}_{1}$ allows us to use Eq. (5) in order to prove that solutions to (1) converge to the similarity profiles in Ref. 1 as $j, t \rightarrow+\infty$. In fact, defining

$$
Q(\omega):=\left(\frac{3}{(1+2 \omega) \alpha}\right)^{\frac{1}{2+\omega}}\left(\frac{2+\omega}{3}\right)^{r} \quad \text { where } r:=\frac{1-\omega}{2+\omega},
$$

the following holds true:

Theorem 2.2. Assume (H1). Let $\left(c_{j}\right)$ be any solution of (1) with initial data $c_{j}(0) \in \ell_{1}$. Let $\varsigma(t)$ and $\widetilde{c}_{j}(\varsigma)$ be as above. Then

(i) $\lim _{\substack{j, \varsigma \rightarrow+\infty \\ \eta=j / \varsigma \text { fixed } \\ \eta \neq 1}} Q(\omega) \varsigma^{r} \widetilde{c}_{j}(\varsigma)= \begin{cases}(1-\eta)^{-r} & \text { if } \eta<1 \\ 0 & \text { if } \eta>1\end{cases}$

(ii) furthermore, if $c_{j}(0)=0$ for $j \geq 2$,

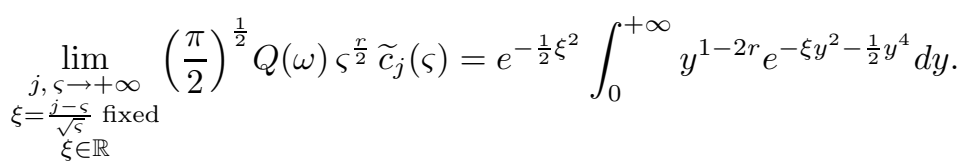

Remark 2.1. The extra assumption on the initial data in Theorem 2.2 (ii) is most likely unnecessary but we are presently unable to overcome it (see Section 6.2 of Ref. 6 for an explanation of the technical problem involved).

In Fig. 1 and 2 we present plots of the similarity limits given in Theorem 2.2 for several values of $\omega$. Note that the profiles in Fig. 2 provide a kind of inner expansion of the jump discontinuities occurring in the profiles in Fig. 1 when $\omega \leq 1$.

The proof of Theorem 2.2 is in no way dependent on the function $\varepsilon(t)$, since it is based on the analysis of Eq. (5) which does not involve any information concerning the input of monomers other than that carried over by the $\widetilde{c}_{1}(\varsigma)$. We direct the interested reader to Refs. 1 and 6 for the proof. 


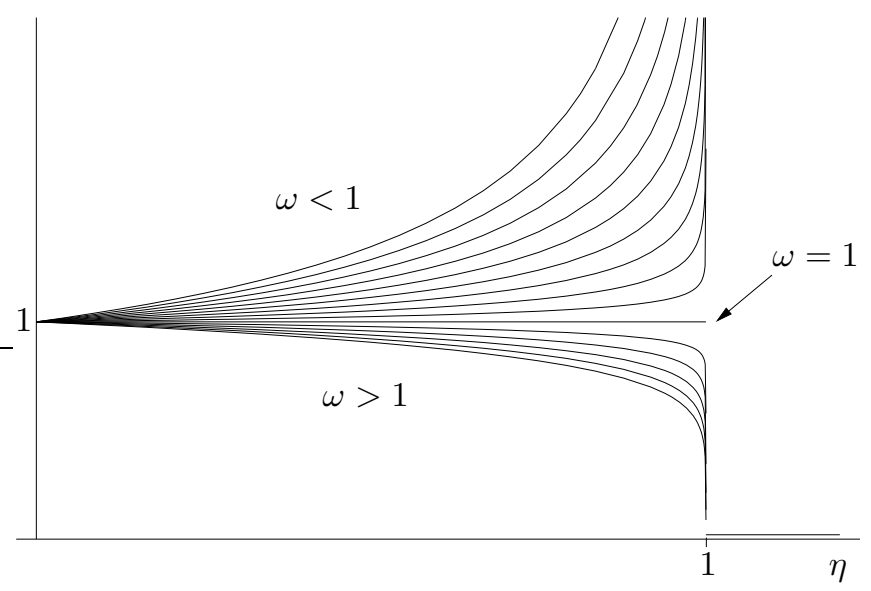

Fig. 1. Graphs of the similarity limits in Theorem 2.2 (i) for values of $\omega$ below and above 1 in steps of 0.1

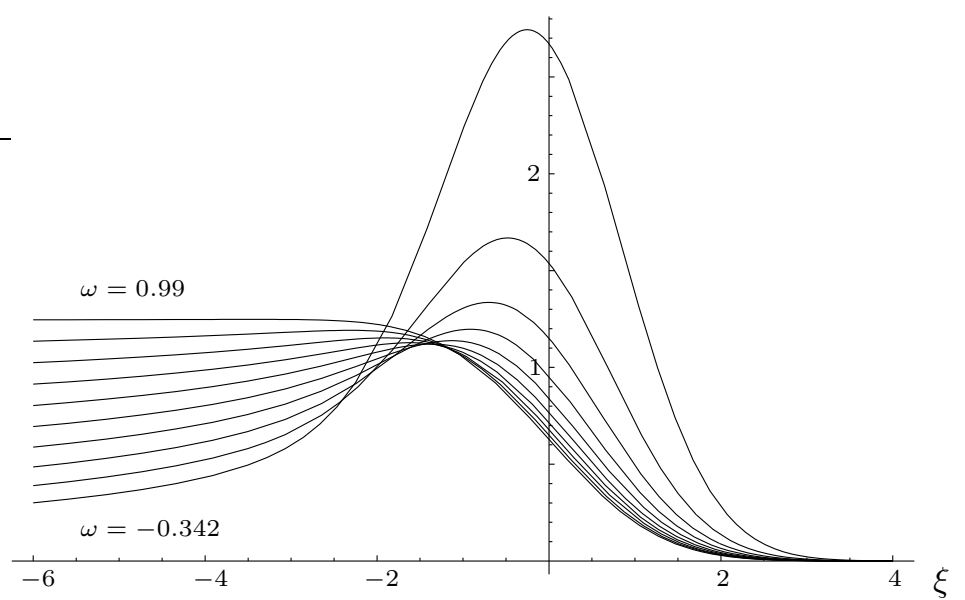

Fig. 2. Graphs of the similarity limits in Theorem 2.2 (ii) for values of $\omega$ from -0.342 to 0.99 in steps of 0.148 .

In the remaining of this note we concentrate on the proof of Theorem 2.1 , and specially in those parts of the proof that differ from the proof of the corresponding result in Ref. 1. 


\section{On the proof of Theorem 2.1}

The general plan of the proof of Theorem 2.1 is analogous to that of the corresponding theorem in Ref. 1: defining a new time scale $t \mapsto \tau$ so that $\frac{d \tau}{d t}=\left(\frac{3 \alpha^{2}}{1+2 \omega}\right)^{1 / 3} t^{\frac{1+2 \omega}{3}}$, letting

$$
\left\{\begin{array}{l}
x(\tau):=\left(\frac{3}{(1+2 \omega) \alpha}\right)^{\frac{1}{3}} t(\tau)^{\frac{1-\omega}{3}} c_{1}(t(\tau)) \\
y(\tau):=\left(\frac{1+2 \omega}{3 \alpha^{2}}\right)^{\frac{1}{3}} t(\tau)^{-\frac{1+2 \omega}{3}} c_{0}(t(\tau)),
\end{array}\right.
$$

and using (H1), system (3) becomes

$$
\left\{\begin{array}{l}
x^{\prime}=(1+\varepsilon(\tau)-x y)-A \tau^{-\frac{1}{2}} x^{2}+B \tau^{-1} x \\
y^{\prime}=(1+\varepsilon(\tau)-x y) \cdot A \tau^{-\frac{1}{2}}-A^{2} \tau^{-1} y,
\end{array}\right.
$$

where $A:=\left(\frac{1+2 \omega}{4+2 \omega}\right)^{\frac{1}{2}}, B:=\frac{1-\omega}{4+2 \omega}$, and, in order not to overload the notation we keep denoting by $\varepsilon(\cdot)$ the function $\varepsilon(t(\cdot))$. To prove Theorem 2.1 it is sufficient to prove that all non-negative solutions $(x, y)$ to system $(6)$ satisfy $(x(\tau), y(\tau)) \rightarrow(1,1)$ as $\tau \rightarrow+\infty$, and in order to obtain this result we may start by proving the positivity and relative boundedness of the solution vector (meaning that $y$ [resp. $x$ ] is bounded iff $x$ [resp. $y$ ] is bounded away from zero); these are proved exactly as in Lemmas 1 and 2 of Ref. 1.

Next we need to prove the boundedness of the orbit $(x(\tau), y(\tau))$ when $\tau \rightarrow+\infty$. This is where things start to look a bit different from what happened in Ref. 1. By (H1) we need only to consider times large enough so that $|\varepsilon(\tau)|<\epsilon$ (where $\epsilon$ can be chosen arbitrarily small) if $\tau>T_{\epsilon}$ say. Observe that from the $y$-equation in system (6) we have $y^{\prime}<0$ if $(x, y) \in$ $\Omega_{\epsilon}^{-}:=\{1+\epsilon-x y<0\}$ and so $y(\tau)$ can only escape to $+\infty$ if the orbit ultimately remains in $\Omega_{\epsilon}^{+}:=\mathbb{R}^{2+} \backslash \Omega_{\epsilon}^{-}$. But to show that this cannot occur an argument similar to the one used in step 1 of the proof of Lemma 3 in Ref. 1 is enough, with the natural changes of the geometrical setting provided by Fig. 3. Without loss of generality we can assume the initial point $P_{\tau_{0}}$ is in $\Omega_{\epsilon}^{+}$with $P_{\tau_{0}}>\max \left\{1, \tau_{L, \epsilon}\right\}$, where $\tau_{L, \epsilon}:=\left(2\left(\frac{L^{2}}{1+\epsilon}-\frac{1+\epsilon}{L}\right)\right)^{-2}$, and $L>\sqrt[3]{1+\epsilon}$ is the ordinate of $P_{\tau_{0}}$.

Using the differential equations (6) we easily conclude that, in the region of Fig. 3 where $y>\frac{L^{2}}{1+\epsilon} x$ and $x<\frac{1+\epsilon}{L}$ the following holds true:

$$
y^{\prime}<\left(x^{\prime}+\left(\frac{1+\epsilon}{L}-\frac{L^{2}}{1+\epsilon}-B A^{-1} \tau^{-\frac{1}{2}}\right) x A \tau^{-\frac{1}{2}}\right) A \tau^{-\frac{1}{2}}<x^{\prime} A \tau^{-\frac{1}{2}}<x^{\prime},
$$

where the last two inequalities hold true for all $\tau>\tau_{0}>\max \left\{1, \tau_{L, \epsilon}\right\}$. But this is the same inequality as in Ref. 1 and from this uniform bound on 


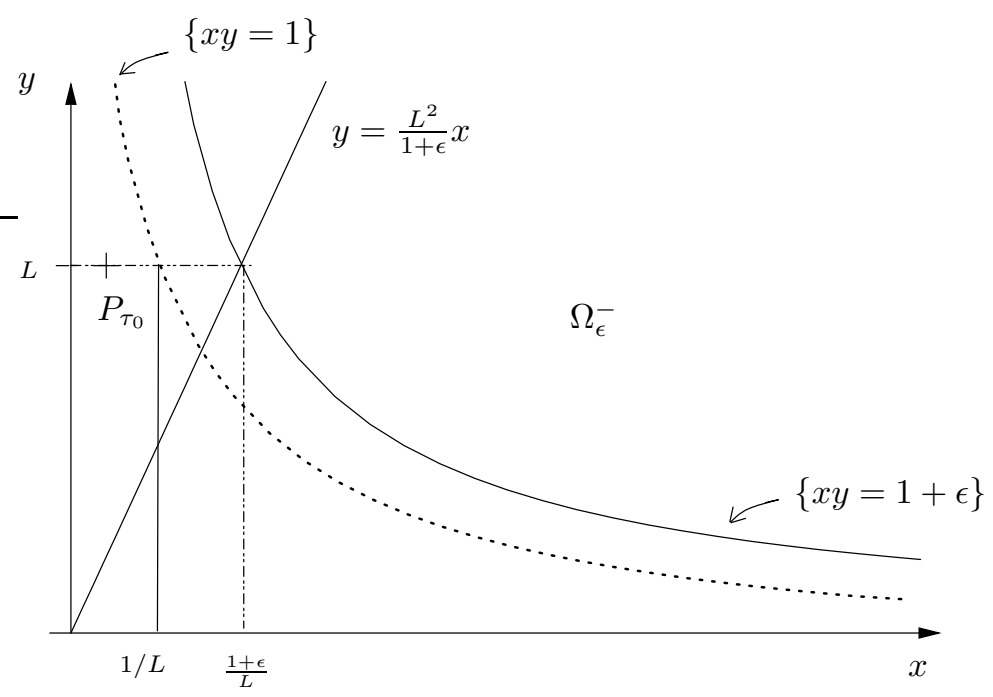

Fig. 3. Geometric setting for the analysis of the possibility of an orbit starting at $P_{\tau_{0}}$ to escape to infinity due to $y(\tau) \rightarrow+\infty$ as $\tau \rightarrow+\infty$.

the slope $\frac{d y}{d x}$ of the orbit, and the behaviour of $y^{\prime}$ in $\Omega_{\epsilon}^{-}$, we immediately conclude $y(\tau)$ cannot diverge to $+\infty$ as $\tau \rightarrow+\infty$. The analysis of what happens for large $x$, and in particular the impossibility for $x(\tau)$ to become unbounded as $\tau \rightarrow+\infty$, requires in the present case an altogether different approach to that used in Ref. 1. There we used a method based on the behaviour of the orbit on the level sets of an auxiliary function approaching the (fixed) hyperbola $\{x y=1\}$. This essentially meant a change to a kind of moving reference system. Now this method is not likely to work in this case without unnatural (and unreasonable) restrictions on the perturbation $\varepsilon(\tau)$. Hence, we now use a novel approach. Let us suppose an orbit of (6) satisfies $x\left(\tau_{n}\right) \rightarrow+\infty$, for some sequence $\tau_{n}$ such that $\tau_{n} \rightarrow+\infty$. Then this orbit must eventually leave $\Omega_{\varepsilon(\tau)}^{-}:=\{1+\varepsilon(\tau)<x y\}$ since otherwise we would have $x^{\prime}=1+\varepsilon(\tau)-x y-A \tau^{-\frac{1}{2}} x^{2}+B \tau^{-1} x<A \tau^{-\frac{1}{2}} x\left(-x+B A^{-1}\right) \tau^{-\frac{1}{2}}<$ 0 for all sufficiently large $\tau$, a contradiction. An easy argument, as the one in Ref. 1 shows that for all large times, the orbit cannot enter $\Omega_{\varepsilon(\tau)}^{-}$. Furthermore, for all large enough $\tau$ it easily follows from Eqs. (6) that

$$
A \tau^{-\frac{1}{2}} x^{\prime}-y^{\prime}=-\left(x-\frac{y}{x}-\frac{B}{A} \tau^{-\frac{1}{2}}\right) A^{2} \tau^{-1} x<0 .
$$

Now suppose $x^{\prime}>0$ for all sufficiently large $\tau$. From (7) this entails $y^{\prime}>$ $A \tau^{-\frac{1}{2}} x^{\prime}>0$, which leads to a contradiction since the orbit would eventually 
enter $\Omega_{\varepsilon(\tau)}^{-}$for large enough $\tau$. So, in order to have $x\left(\tau_{n}\right) \rightarrow+\infty$ as $\tau_{n} \rightarrow$ $+\infty$, there must exist an infinite sequence of time intervals in which $x^{\prime}<0$, and in at least some subintervals we must have $y^{\prime}<0$, since otherwise the orbit would enter in $\Omega_{\varepsilon(\tau)}^{-}$anyway, or it would remain bounded. The differential inequality (7) implies that the situation depicted in Fig. 4 is essentially the only possible one.

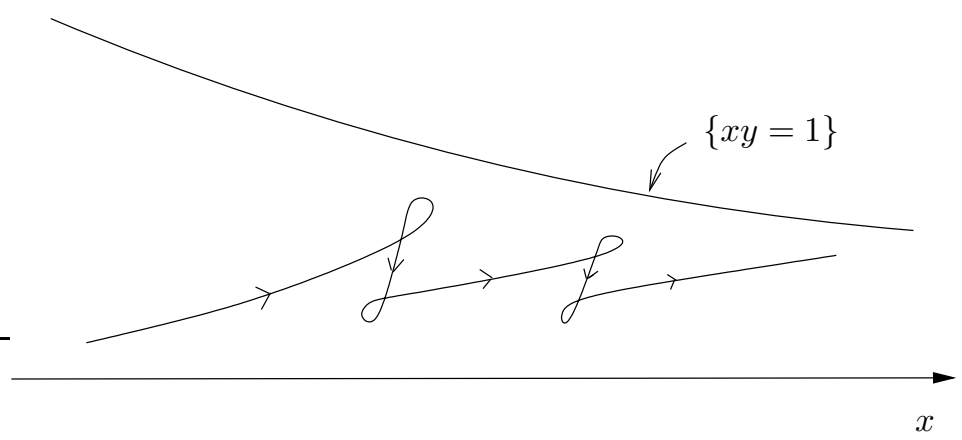

Fig. 4. The only possibility to have an orbit with $x(\tau) \rightarrow+\infty$ as $\tau \rightarrow+\infty$. Note that (7) implies that $y^{\prime}(\tau)>0$ whenever $x^{\prime}(\tau)>0$

Observe that for the orbit to decrease in the $y$ component it must first start decreasing in $x$, since (7) forces $A \tau^{-\frac{1}{2}} x^{\prime}<y^{\prime}$. This explains why the orbit should essentially be as shown in Fig. 4, with that type of selfcrossings. But this is impossible. To see why, let us concentrate our attention in Fig. 5, where a portion of the orbit of Fig. 4 at the start of one of its $y$-descent sections is enlarged showing a self-crossing $P$ that occurs at two instants, $\tau_{1}$ and $\tau_{2}$, with $\tau_{1}<\tau_{2}$.

At $\tau_{1}$ we have $x^{\prime}\left(\tau_{1}\right)>0$ and $(7)$ gives $\frac{d y}{d x}\left(\tau_{1}\right)>A \tau_{1}^{-\frac{1}{2}}$, and at $\tau_{2}$ we have $x^{\prime}\left(\tau_{2}\right)<0$, which implies that (7) now means $\frac{d y}{d x}\left(\tau_{2}\right)<A \tau_{2}^{-\frac{1}{2}}$. But since $\tau_{2}>\tau_{1}$, we conclude that $\frac{d y}{d x}\left(\tau_{2}\right)<A \tau_{2}^{-\frac{1}{2}}<A \tau_{1}^{-\frac{1}{2}}<\frac{d y}{d x}\left(\tau_{1}\right)$ in contradiction with what we concluded above should happen at a selfcrossing point. This concludes the proof that $(x, y)$ must remain bounded (and bounded away from zero).

With this information we can now start to identify the orbit's $\omega$-limit, by showing that the $\omega$-limit set of any orbit is contained in the hyperbola $\{x y=1\}$. In order to obtain this result we consider an auxiliary function $h(\tau):=x(\tau) y(\tau)$; as was done in Ref. 1, if $(x, y)$ satisfies $(7)$ then $h(\tau)$ 

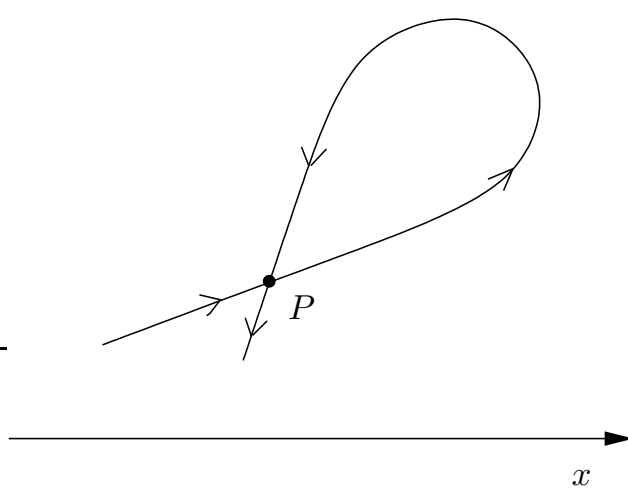

Fig. 5. Situation described in the text for a self-intersecting point of an orbit having $x(\tau) \rightarrow+\infty$ as $\tau \rightarrow+\infty$.

satisfies the following linear (in $h$ ) differential equation:

$$
h^{\prime}(\tau)=(1+\varepsilon(\tau))\left(y+A \tau^{-\frac{1}{2}} x\right)-\alpha(\tau) h(\tau),
$$

where

$$
\alpha(\tau):=y(\tau)+2 A \tau^{-\frac{1}{2}} x(\tau)+\left(A^{2}-B\right) \tau^{-1} .
$$

Using the variation of constants formula we can write the solution to (8) as

$$
h(\tau)=h\left(\tau_{0}\right) e^{-\int_{\tau_{0}}^{\tau} \alpha(s) d s}+\int_{\tau_{0}}^{\tau}(1+\varepsilon(s))\left(y(s)+\frac{A x(s)}{s^{\frac{1}{2}}}\right) e^{-\int_{s}^{\tau} \alpha(\theta) d \theta} d s .
$$

We need to show that $h \rightarrow 1$. Using Eq. (10), we show that the first term goes to zero and that the second one goes to 1 , as $\tau \rightarrow+\infty$. Since $y$ is bounded away from zero, there exists a constant $L_{y}>0$ such that $y(\tau) \geq$ $L_{y}$, and so

$$
\begin{aligned}
e^{-\int_{\tau_{0}}^{\tau} \alpha(s) d s} & =\left(\frac{\tau_{0}}{\tau}\right)^{A^{2}-B} e^{-\int_{\tau_{0}}^{\tau}\left(y(s)+2 A s^{-1 / 2} x(s)\right) d s} \\
& \leq\left(\frac{\tau_{0}}{\tau}\right)^{A^{2}-B} e^{-\int_{\tau_{0}}^{\tau} y(s) d s} \leq\left(\frac{\tau_{0}}{\tau}\right)^{A^{2}-B} e^{-L_{y}\left(\tau-\tau_{0}\right)},
\end{aligned}
$$

showing that the first term in Eq. (10) goes to zero (exponentially) as $\tau \rightarrow+\infty$.

Next we show that the second term in Eq. (10) goes to 1 as $\tau \rightarrow+\infty$. Using again the fact that $|\varepsilon(\tau)|<\epsilon$ if $\tau>T_{\epsilon}$, we can estimate the second term in Eq. (10) by splitting the integral as $\int_{\tau_{0}}^{\tau}=\int_{\tau_{0}}^{T_{\epsilon}}+\int_{T_{\epsilon}}^{\tau}$. The integral 
over $\left[\tau_{0}, T_{\epsilon}\right]$ converge to zero as $\tau \rightarrow \infty$, since we are integrating an exponentially decaying function on a fixed compact interval. On $\left[T_{\epsilon}, \tau\right]$, we can write

$$
(1-\epsilon) \int_{T_{\epsilon}}^{\tau} g(s, \tau) d s \leq \int_{T_{\epsilon}}^{\tau}(1+\varepsilon(\tau)) g(s, \tau) d s \leq(1+\epsilon) \int_{T_{\epsilon}}^{\tau} g(s, \tau) d s .
$$

where $g(s, \tau):=\left(y(s)+A s^{-\frac{1}{2}} x(s)\right) e^{-\int_{s}^{\tau} \alpha(\theta) d \theta}$. Using the results from the proof of Lemma 4 in Ref. 1 we have $\int_{T_{\epsilon}}^{\tau} g(s, \tau) d s \rightarrow 1$ as $\tau \rightarrow+\infty$ and this completes the proof of the convergence of $h$.

Inspired by the approach in Ref. 1 , the next steps to locate the $\omega$-limit set of the orbits will be, first to prove that, for each orbit, its $\omega$-limit set, (which, by the previous result about $h$, is an arc of the hyperbola $\{x y=1\}$ ) contains the point $(1,1)$, and second to conclude that that arc degenerates into the single point $(1,1)$. Resorting, as in Ref. 1 , to the auxiliary function $b(\tau):=y(\tau)-A \tau^{-\frac{1}{2}} x(\tau)$ we discover that its dynamics is governed by the differential equation

$$
b^{\prime}=\left(-b+x^{2}\right) A^{2} \tau^{-1},
$$

which is exactly the same as in Ref. 1, i.e., the dynamic behaviour of $b$ is independent of $\varepsilon(\tau)$ and so all the results proved when $\varepsilon(\tau) \equiv 0$ in Lemmas 5 and 6 of Ref. 1 remain valid for the present case. Hence the two steps referred to above can be completed and the proof of Theorem 2.1 is achieved.

\section{Remarks on the Rate of Convergence}

A question that naturally comes to mind at this point is to ask at what rate do solutions converge to their final states. Whatever the mathematical sense we may give this question, its elucidation is bound to include the long time behaviour of the quantity $J_{1}(t)-c_{0}(t) c_{1}(t)$ (if we are thinking in terms of solutions to $(3)$ ) or of $1+\varepsilon(\tau)-x(\tau) y(\tau)$ (if we are considering solutions to (6).)

In the case $\varepsilon(t) \equiv 0$ treated in Ref. 1 the assumptions in Theorem 2.1 are sufficient to prove that

$$
\frac{1-x(\tau) y(\tau)}{A \tau^{-\frac{1}{2}}} \longrightarrow 1 \quad \text { as } \quad \tau \rightarrow+\infty
$$

and hence to have, as $t \rightarrow+\infty$,

$$
\left(\frac{3}{(1+2 \omega) \alpha}\right)^{\frac{2}{3}} t^{2 \frac{1-\omega}{3}}\left(\alpha t^{\omega}-c_{0}(t) c_{1}(t)\right) \longrightarrow 1 \text {. }
$$


In order to obtain similar results when $\varepsilon(t)$ is not identically zero we need further assumptions on this function besides those stated in (H1), namely we need to assume something about its decay rate to zero. The following hypothesis is sufficient to ensure (12) holds also in this case.

(H2) The function $\varepsilon(t)$ is continuously differentiable and, as $t \rightarrow+\infty$, satisfies $\varepsilon(t)=\mathcal{O}\left(t^{-\frac{2+\omega}{3}}\right)$, and $\dot{\varepsilon}(t)=\mathcal{O}\left(t^{-\frac{1-\omega}{3}}\right)$.

Remark 4.1. Observe that, by the definition of the time scale $\tau$ given in the beginning of Section 3, we easily conclude that $\tau^{-\frac{1}{2}}=\mathcal{O}\left(t^{-\frac{2+\omega}{3}}\right)$, from which it follows that we must have $\varepsilon(\tau)=\mathcal{O}\left(\tau^{-\frac{1}{2}}\right)$ and also $\varepsilon^{\prime}(\tau)=\dot{\varepsilon} \frac{d t}{d \tau}=$ $\mathcal{O}\left(\tau^{-\frac{1}{2}}\right)$.

The way the proof of (11) proceeds is analogous to the proof of Lemmas 4 and 7 in Ref. 1: in the present case the expression for $(1-h(\tau)) / \tau^{-1 / 2}$ has the additional additive contribution coming from the perturbation term $\varepsilon(\tau)$, namely

$$
-\tau^{\frac{1}{2}} \int_{\tau_{0}}^{\tau} \varepsilon(s)\left(y(s)+A s^{-\frac{1}{2}} x(s)\right) e^{-\int_{s}^{\tau} \alpha(\theta) d \theta} d s,
$$

with $\alpha(\cdot)$ the function defined in (9). The analysis of (13) proceeds as follows: first write it as

$-\tau^{\frac{1}{2}} \int_{\tau_{0}}^{\tau} \varepsilon(s) \alpha(s) e^{-\int_{s}^{\tau} \alpha(\theta) d \theta} d s+\tau^{\frac{1}{2}} \int_{\tau_{0}}^{\tau} \varepsilon(s)\left(\frac{A x(s)}{s^{1 / 2}}+\frac{A^{2}-B}{s}\right) e^{-\int_{s}^{\tau} \alpha(\theta) d \theta} d s$,

then observe that the first integral in (14) can be written as follows

$$
\begin{aligned}
\int_{\tau_{0}}^{\tau} \varepsilon(s) \alpha(s) e^{-\int_{s}^{\tau} \alpha(\theta) d \theta} d s & =e^{-\int_{\tau_{0}}^{\tau} \alpha(s) d s} \int_{\tau_{0}}^{\tau} \varepsilon(s) \frac{d}{d s}\left(e^{\int_{\tau_{0}}^{s} \alpha(\theta) d \theta}\right) d s \\
& =\varepsilon(\tau)-\varepsilon\left(\tau_{0}\right) e^{-\int_{\tau_{0}}^{\tau} \alpha(s) d s}-\int_{\tau_{0}}^{\tau} \varepsilon^{\prime}(s) e^{\int_{\tau_{0}}^{s} \alpha(\theta) d \theta} d s
\end{aligned}
$$

where the last equality was obtained using integration by parts. Now, the estimates developed in the proof of Lemma 4 of Ref. 1 together with the assumption (H2), in the version presented in Remark 4.1, allow us to control the second integral in (14) and the right-hand side of (15), thus completing the proof of (11), and thus also the corresponding version of (12) that now reads as

$$
\left(\frac{3}{(1+2 \omega) \alpha}\right)^{\frac{2}{3}} t^{2 \frac{1-\omega}{3}}\left((1+\varepsilon(t)) \alpha t^{\omega}-c_{0}(t) c_{1}(t)\right) \underset{t \rightarrow+\infty}{\longrightarrow} 1
$$




\section{Final Remarks}

In this note we considered the addition model Eq. (1) with power-like timedependent input of monomers (i.e., addition of monomers at a rate satisfying (H1)). We proved that, considering the long time and cluster size limit with either $\eta:=j / \varsigma$ or $\xi:=(j-\varsigma) / \sqrt{\varsigma}$ constant (where $\varsigma$ is an appropriate rescaling of the original time variable), the solutions approach universal profiles, independently of the initial data. This type of convergence, referred to in the literature as self-similar behaviour, has been the subject of a number of recent studies in the context of Smoluchowski coagulation systems (cf. Refs. 2, 3, 4). The present study extends our knowledge of such a behaviour in Beker-Döring like coagulation equations, by complementing recent results, obtained for constant (Ref. 6) and for particular time-dependent (Ref. 1) inputs of monomers, to a much larger class of timeindependent inputs. Since our results include the cases where the input rate of monomers can have a rather erratical behaviour (albeith continuous and asymptotically approaching a power $\alpha t^{\omega}$, with $\omega>-\frac{1}{2}$ ), it provides a further indication of the robustness of the convergence to self-similar behaviour in this class of systems.

\section{References}

1. F. P. da Costa and R. Sasportes, Dynamics of a non-autonomous ODE system occurring in coagulation theory, J. Dynam. Differential Equations, published on-line: 3 January 2007, DOI: 10.1007/s10884-006-9067-5.

2. F. P. da Costa, M. Grinfeld, W. Lamb and J. A. D. Wattis (eds.), CoagulationFragmentation Processes, Physica D 222, pp. 1-166 (2006) (special issue).

3. Ph. Laurençot and S. Mischler, On Coalescence Equations and Related Models, in Modelling and Computational Methods for Kinetic Equations, Eds. P. Degond, L. Pareschi, G. Russo (Birkhäuser, Boston, 2004) pp. 321-356.

4. F. Leyvraz, Scaling theory and exactly solved models in the kinetics of irreversible aggregation, Phys. Rep. 383, 95 (2003).

5. J. G. Amar, M. N. Popescu, F. Family, Rate-equation approach to island capture zones and size distributions in epitaxial growth, Phys. Rev. Letters 86, 3092 (2001).

6. F. P. da Costa, H. van Roessel and J. A. D. Wattis, Long-time behaviour and self-similarity in a coagulation equation with input of monomers, Markov Processes Relat. Fields 12, 367 (2006).

7. J. A. D. Wattis, Similarity solutions of a Becker-Döring system with timedependent monomer input, J. Phys. A: Math. Gen. 37, 7823 (2004). 